\title{
From snakes to region-based active contours defined by region-dependent parameters
}

\author{
Stéphanie Jehan-Besson ${ }^{1}$, Muriel Gastaud ${ }^{1}$, Frédéric Precioso ${ }^{1}$, \\ Michel Barlaud $^{1}$, Gilles Aubert ${ }^{2}$, Éric Debreuve ${ }^{1}$ \\ ${ }^{1}$ Laboratoire I3S, UMR CNRS 6070, Les Algorithmes, Bât. Euclide B, \\ 2000, route des lucioles, BP 121, 06903 Sophia Antipolis Cedex, France \\ ${ }^{2}$ Laboratoire Dieudonné, UMR CNRS 6621, Université de Nice-Sophia Antipolis, \\ Parc Valrose, 06108 Nice Cedex 2, France
}

barlaud@i3s.unice.fr

This synthetic paper deals with image and sequence segmentation when looking at the segmentation task from a criterion optimization point of view. Such a segmentation criterion involves so-called (boundary and region) descriptors which, in the general case, may depend on their respective boundary or region. This dependency must be taken into account when computing the criterion derivative with respect to the unknown object domain (defined by its boundary). If not, some correctional terms may be omitted. This article focuses computing the derivative of the segmentation criterion using a dynamic scheme. The presented scheme is general enough to provide a framework for a wide variety of applications in segmentation. It also provides a theoretical meaning to the active contour philosophy. (c) 2003 Optical Society of America

\section{Introduction}

This paper does the synthesis of several years of development in active contour segmentation conducted at I3S (Informatique, Signaux et Systèmes de Sophia Antipolis) and Dieudonné laboratories, CNRS (French National Research Center) and University Of Nice-Sophia Antipolis, France.

The purpose of segmentation is to isolate an object (or several objects) of interest in an image or a sequence. Given an initial contour (a closed curve), the active contour technique consists in applying locally a force (or displacement, or velocity) such that the initial contour evolves toward the contour of the object of interest. This force is derived from a characterization of the object formally written as a criterion to be optimized.

\section{A. Boundary-Based Active Contours}

In boundary-based active contour techniques, the object is characterized by properties of its contour only. The original active contour developments were called snakes. ${ }^{1}$ Only the convex hull of objects could be segmented because these techniques were based 
on a minimum length penalty. In order to be able to segment concave objects, a balloon force was heuristically introduced. It was later theoretically justified as a minimum area constraint ${ }^{2}$ balancing the minimum length constraint. The geodesic active contour technique ${ }^{3}$ is the most general form of boundary-based techniques. The criterion corresponding to this technique is

$$
J(\Gamma)=\int_{\Gamma} k(x) \mathrm{d} x
$$

where $\Gamma$ is a contour, $k$ is a positive function "describing" the object of interest, and $x$ is a point of the image. The active contour evolution equation ${ }^{3}$ is

$$
\frac{\partial \Gamma}{\partial \tau}=(\kappa k-\nabla k \cdot \boldsymbol{N}) \boldsymbol{N}
$$

where $\tau$ is the evolution parameter $\left(\Gamma(\tau=0)=\Gamma_{0}\right.$, initial contour, and $\Gamma(\tau \rightarrow \infty) \rightarrow$ segmentation), $\kappa$ is the curvature of $\Gamma$, operator $\cdot$ represents the inner product, and $\boldsymbol{N}$ is the inward normal. Actually, this equation should be written with $\kappa(x), k(x)$, and $\boldsymbol{N}(x)$ where $x$ is a point of $\Gamma(\tau)$. The contour minimizing $J$ can be interpreted as the curve of minimum length in the metric defined by function $k$. If $k$ is the constant function equal to one, Eq. (2) is called the geometric heat equation by analogy with the heat diffusion equation. Function $k$ can also be a function of the gradient of the image. For instance,

$$
k(x)=\frac{1}{1+|\nabla I(x)|}
$$

where $I$ is the image and $\nabla$ is the intensity gradient. In this case, the object contour is simply characterized by a curve following high gradients. As a consequence, the technique is effective only if the contrast between the object and the background is high. Moreover, high gradients in an image may correspond to the boundaries of objects that are not of interest. Regardless of function $k$, information on the boundary is too local for segmentation of complex scenes. A global, more sophisticated object characterization is needed.

\section{B. Region-Based Active Contours}

In order to better characterize an object and to be less sensitive to noise, region-based active contour techniques were proposed. ${ }^{4,5}$ A region is represented by mathematical expressions called "descriptors" in this paper. Two kinds of region are usually considered: The object of interest and the background. Note that region-based/boundarybased hybrid techniques are common. ${ }^{6-9}$ In the general case, descriptors may depend on their respective regions, for instance, statistical features such as mean intensity or variance within the region. ${ }^{10}$ The general form of a criterion including both regionbased and boundary-based terms is

$$
J\left(\Omega_{\text {in }}\right)=\int_{\Omega_{\text {in }}} k_{\text {in }}\left(\Omega_{\text {in }}, x\right) \mathrm{d} x+\eta \int_{\Gamma} k_{b}(x) \mathrm{d} x
$$


where $\Omega_{\text {in }}$ is the domain inside $\Gamma$ and $\eta$ is a positive constant. An example of descriptor $k_{\text {in }}$ is

$$
k_{\text {in }}\left(\Omega_{\text {in }}, x\right)=\left(\mu\left(\Omega_{\text {in }}\right)-I(x)\right)^{2}
$$

where $\mu$ is the mean intensity

$$
\mu\left(\Omega_{\mathrm{in}}\right)=\frac{\int_{\Omega_{\mathrm{in}}} I(x) \mathrm{d} x}{\int_{\Omega_{\mathrm{in}}} \mathrm{d} x} .
$$

Function (3) is an example of descriptor $k_{b}$. Classically the integral on domain $\Omega_{\text {in }}$ in criterion (4) is reduced to an integral along $\Gamma$ using the Green-Riemann theorem $^{6-8,11-13}$ or continuous media mechanics techniques. ${ }^{14,15}$ Two active contour approaches are possible to minimize the resulting criterion: (i) It is possible to determine the evolution of an active contour from $\tau$ to $\tau+d \tau$ without computing a velocity: The displacement of a point of the contour is chosen among small random displacements as the one leading to the locally optimal criterion value. ${ }^{6,11}$ However this implies to compute the criterion value several times for each point; (ii) Alternatively, differentiating the criterion with respect to $\tau$ allows to find an expression of the appropriate displacement, or velocity, for each point. ${ }^{7,8,12,13}$ In this case the region-dependency of the descriptors must be taken into account. If not, ${ }^{7,8,12-15}$ some correctional terms in the expression of the velocity may be omitted.

\section{Accounting For Region Dependency}

In this paper, the region-dependency of descriptors is taken into account in the derivation of the velocity. It is shown that it induces additional terms leading to a greater accuracy of segmentation. The development is general enough to provide a framework for region-based active contour. It is inspired by shape optimization techniques. ${ }^{16,17}$

\section{A. Problem Statement}

Let us consider an image composed of background and one object of interest, each of which having unique properties represented by descriptors $k_{\text {out }}$ and $k_{\text {in }}$, respectively. The object boundary has also properties represented by descriptor $k_{b}$. For a given domain $\Omega_{\text {in }}$, let us define the following general criterion

$$
J\left(\Omega_{\text {in }}\right)=\alpha \int_{\Omega_{\text {in }}} k_{\text {in }}\left(\Omega_{\text {in }}, x\right) \mathrm{d} x+\beta \int_{\Omega_{\text {out }}} k_{\text {out }}\left(\Omega_{\text {out }}, x\right) \mathrm{d} x+\int_{\Gamma} k_{b}(x) \mathrm{d} x
$$

where $\alpha$ and $\beta$ are positive constants, $\Omega_{\text {in }}, \Omega_{\text {out }}$, and $\Gamma$ are such that

$$
\left\{\begin{array}{l}
\Omega_{\text {in }} \cup \Gamma \cup \Omega_{\text {out }}=\text { image domain } D \\
\Gamma=\partial \Omega_{\text {in }}=\partial \Omega_{\text {out }} \\
\Omega_{\text {in }}=\text { domain inside } \Gamma
\end{array},\right.
$$

and $k_{\text {in }}, k_{\text {out }}$, and $k_{b}$ are positive functions such that

$$
\left\{\begin{array}{l}
k_{\text {in }} \text { is minimum in the object, e.g., function }(5) \\
k_{\text {out }} \text { is minimum in the background, e.g., } k_{\text {out }}=0, \\
\text { meaning that no specific information is available for the background } \\
k_{b} \text { is minimum on the object boundary, e.g., function }(3)
\end{array}\right. \text {. }
$$


Therefore, the minimum of criterion (7) is reached if $\Omega_{\text {in }}$ segments the object of interest. The proposed segmentation method is based on the active contour technique (see Subsection 2.B below). Let us give the notations here. Domains $\Omega_{\text {in }}$ and $\Omega_{\text {out }}$ and contour $\Gamma$ are respectively replaced with dynamic versions depending on evolution parameter $\tau$. At $\tau$ equal to zero, an initial domain $\Omega_{\text {in }}(\tau=0)=\Omega_{\text {in }}^{0}$ is defined, either manually or automatically (equivalently, $\Omega_{\text {out }}(\tau=0)=\Omega_{\text {out }}^{0}$ and $\left.\Gamma(\tau=0)=\Gamma_{0}\right)$. The active contour converges toward the object boundary as $\tau$ increases. It increases by $d \tau$ at each iteration when discretizing the evolution equation for computer coding.

\section{B. Differentiating the Criterion}

The set of domains $\Omega_{\text {in }}$ is not a vectorial space. Direct computation of the derivative of criterion (7) with respect to $\Omega_{\text {in }}$ is not possible. The proposed solution is to use a dynamic scheme where $\Omega_{\text {in }}$ becomes continuously dependent on an evolution parameter denoted by $\tau$. As $\tau$ increases, $\Omega_{\text {in }}(\tau)$ must act as a minimizing sequence of criterion (7). It is equivalent to finding a family of domain transforms $T_{\tau}$ such that $T_{0}$ is the identity and $T_{\tau}\left(\Omega_{\text {in }}^{0}\right)=\Omega_{\text {in }}(\tau)$ where $\Omega_{\text {in }}^{0}$ is an initial contour. As a consequence, the active contour philosophy of the proposed method has a theoretical explanation rather than being used as an implementation tool for an energy minimization problem. Criterion (7) becomes

$$
J\left(\Omega_{\text {in }}(\tau)\right)=\alpha \int_{\Omega_{\text {in }}(\tau)} k_{\text {in }}\left(\Omega_{\text {in }}(\tau), x\right) \mathrm{d} x+\beta \int_{\Omega_{\text {out }}(\tau)} k_{\text {out }}\left(\Omega_{\text {out }}(\tau), x\right) \mathrm{d} x+\int_{\Gamma(\tau)} k_{b}(x) \mathrm{d} x
$$

which can be rewritten

$$
J(\tau)=\alpha \int_{\Omega_{\text {in }}(\tau)} k_{\text {in }}(\tau, x) \mathrm{d} x+\beta \int_{\Omega_{\text {out }}(\tau)} k_{\text {out }}(\tau, x) \mathrm{d} x+\int_{\Gamma(\tau)} k_{b}(x) \mathrm{d} x
$$

Criterion (11) is composed of two types of integrals:

$$
\begin{aligned}
& J_{1}(\tau)=\int_{\Omega(\tau)} k(\tau, x) \mathrm{d} x \quad \text { (region integral) } \\
& J_{2}(\tau)=\int_{\Gamma(\tau)} k_{b}(x) \mathrm{d} x \quad \text { (contour integral) }
\end{aligned}
$$

For simplicity, $k(\tau, x)$ will be written $k$ and $k_{b}(x)$ will be written $k_{b}$.

\section{Region Integral}

Theorem $1^{16,17}$ Let $D$ be the image domain $(\Omega(\tau) \subset D)$. Let $k$ be a smooth function on $R^{+} \times \bar{D}$. Then

$$
\frac{d J_{1}}{d \tau}(\tau)=J_{1}^{\prime}(\tau)=\int_{\Omega(\tau)} \frac{\partial k}{\partial \tau} d x-\int_{\partial \Omega(\tau)} \boldsymbol{v} \cdot \boldsymbol{N} k d x
$$

where $\boldsymbol{v}$ (actually $\boldsymbol{v}(\tau, x)$ ) is the velocity of $\partial \Omega(\tau)$ and $\boldsymbol{N}$ (actually $\boldsymbol{N}(\tau, x)$ ) is the inward unit normal to $\partial \Omega(\tau)$. 
$J_{1}^{\prime}(\tau)$ is called the Eulerian derivative of $J_{1}(\Omega(\tau))$ in the direction of $\boldsymbol{v}$ at $\tau$. It represents the variation of $J_{1}(\tau)$ due to both the deformation of integration domain $\Omega(\tau)$ according to $\boldsymbol{v}$ and the variation of $k$. The variation of $k$ is also due to the deformation of domain $\Omega(\tau)$.

Corollary $1^{18}$ Let $D$ be the image domain. Let $\Omega_{\mathrm{in}}(\tau), \Omega_{\mathrm{out}}(\tau)$, and $\Gamma(\tau)$ be two domains and a boundary as defined by Eqs. (8). Let $k_{\mathrm{in}}$, respectively $k_{\mathrm{out}}$, be a smooth function on $R^{+} \times \Omega_{\text {in }}(\tau)$, respectively $R^{+} \times \Omega_{\text {out }}(\tau)$. Let $\tilde{J}_{1}(\tau)$ be

$$
\tilde{J}_{1}(\tau)=\int_{\Omega_{\mathrm{in}}(\tau)} k_{\mathrm{in}}(\tau, x) d x+\int_{\Omega_{\mathrm{out}}(\tau)} k_{\mathrm{out}}(\tau, x) d x
$$

Then

$$
\begin{aligned}
\frac{d \tilde{J}_{1}}{d \tau}(\tau) & =\tilde{J}_{1}^{\prime}(\tau) \\
& =\int_{D} \frac{\partial K}{\partial \tau} d x-\int_{\Gamma(\tau)} \boldsymbol{v} \cdot \boldsymbol{N}[[K]] d x-\int_{\partial \Omega_{\text {out }}(\tau) \backslash \Gamma(\tau)} \boldsymbol{w} \cdot \boldsymbol{N}_{\Omega_{\text {out }}} K d x
\end{aligned}
$$

where $K$ is the function equal to $k_{\text {in }}$ in $\Omega_{\text {in }}(\tau)$ and $k_{\text {out }}$ in $\Omega_{\text {out }}(\tau)$, [[K]] is the jump of $K$ across $\Gamma(\tau)$ and it is equal to $k_{\text {in }}-k_{\text {out }}, \partial \Omega_{\text {out }}(\tau) \backslash \Gamma(\tau)$ is the boundary of $\Omega_{\text {out }}(\tau)$ excluding $\Gamma(\tau)$ (namely $\partial D$ ), $\boldsymbol{w}$ is the velocity of $\partial \Omega_{\text {out }}(\tau) \backslash \Gamma(\tau)$, and $\boldsymbol{N}_{\Omega_{\text {out }}}$ is the inward unit normal to $\partial \Omega_{\text {out }}(\tau) \backslash \Gamma(\tau)$.

Corollary 1 is obtained by applying theorem 1 with $\Omega_{\text {in }}(\tau), k_{\text {in }}$ and $D=\Omega_{\text {in }}(\tau)$ for the first integral of (15) and with $\Omega_{\text {out }}(\tau), k_{\text {out }}$ and $D=\Omega_{\text {out }}(\tau)$ for the second integral (see Appendix). Note that the image domain being fixed, velocity $\boldsymbol{w}$ is equal to zero and so is the last integral of (16).

\section{Contour Integral}

The derivative of $J_{2}(\tau)$ is classical. ${ }^{3}$

$$
\frac{d J_{2}}{d \tau}(\tau)=J_{2}^{\prime}(\tau)=\int_{\Gamma(\tau)}\left(\nabla k_{b} \cdot \boldsymbol{N}-k_{b} \kappa\right) \boldsymbol{v} \cdot \boldsymbol{N} \mathrm{d} x
$$

where $\kappa$ (actually $\kappa(\tau, x))$ is the curvature of $\Gamma(\tau)$.

\section{Complete Criterion}

Finally, the derivative of criterion (11) is

$$
\begin{aligned}
J^{\prime}(\tau)= & \alpha \int_{\Omega_{\text {in }}(\tau)} \frac{\partial k_{\text {in }}}{\partial \tau} \mathrm{d} x+\beta \int_{\Omega_{\text {out }}(\tau)} \frac{\partial k_{\text {out }}}{\partial \tau} \mathrm{d} x+ \\
& \int_{\Gamma(\tau)}\left(\beta k_{\text {out }}-\alpha k_{\text {in }}+\nabla k_{b} \cdot \boldsymbol{N}-k_{b} \kappa\right) \boldsymbol{v} \cdot \boldsymbol{N} \mathrm{d} x .
\end{aligned}
$$


The first two integrals in (18) can be reduced to a boundary integral: ${ }^{18}$

$$
\alpha \int_{\Omega_{\text {in }}(\tau)} \frac{\partial k_{\text {in }}}{\partial \tau} \mathrm{d} x+\beta \int_{\Omega_{\text {out }}(\tau)} \frac{\partial k_{\text {out }}}{\partial \tau} \mathrm{d} x=\int_{\Gamma(\tau)} H\left(k_{\text {in }}, k_{\text {out }}\right) \boldsymbol{v} \cdot \boldsymbol{N} \mathrm{d} x
$$

where $H\left(k_{\text {in }}, k_{\text {out }}\right)$ represents the additional terms mentioned at the beginning of section 2. As a consequence, derivative (18) can be rewritten

$$
J^{\prime}(\tau)=\int_{\Gamma(\tau)} \rho \boldsymbol{v} \cdot \boldsymbol{N} \mathrm{d} x
$$

with

$$
\rho=H\left(k_{\text {in }}, k_{\text {out }}\right)+\beta k_{\text {out }}-\alpha k_{\text {in }}+\nabla k_{b} \cdot \boldsymbol{N}-k_{b} \kappa .
$$

Velocity $\boldsymbol{v}$ is unknown. It must be chosen so that the value of (20) is negative in order to make criterion (11) decrease. Note that this result can be obtained by a classical calculus of variation approach although the development is tedious. ${ }^{19}$

\section{Evolution Equation}

A way to ensure negativity of derivative (20) is to chose $\boldsymbol{v}=-\rho \boldsymbol{N}$. Derivative (20) is then

$$
J^{\prime}(\tau)=-\int_{\Gamma(\tau)} \rho^{2} \mathrm{~d} x
$$

Since velocity $\boldsymbol{v}$ can also be written $\frac{\partial \Gamma}{\partial \tau}$, the evolution equation of the active contour is

$$
\left\{\begin{array}{l}
\Gamma(0)=\Gamma_{0} \\
\frac{\partial \Gamma}{\partial \tau}(\tau, x)=-\rho(\tau, x) \boldsymbol{N}(\tau, x) \text { for all } \tau \geq 0 \text { and } x \in \Gamma(\tau)
\end{array} .\right.
$$

Equation (23) means that, starting from initial contour $\Gamma_{0}$, active contour $\Gamma$ evolves until velocity amplitude $\rho$ is equal to zero everywhere along the contour. In other words, active contour $\Gamma$ at $\tau+d \tau$ is obtained by deforming active contour $\Gamma$ at $\tau$ according to local velocity $-\rho \boldsymbol{N}$, if it is not equal to zero. If region descriptors $k_{\text {in }}$ and $k_{\text {out }}$ are region-independent (i.e., if they do not depend on $\left.\Omega_{\text {in }}(\tau)\right)$, then $H\left(k_{\text {in }}, k_{\text {out }}\right)$ is equal to zero. However this is not a necessary condition. Note that Eq. (23) solves the segmentation problem (the primary goal) and computes the parameters involved in the descriptors simultaneously. These parameters could be used for indexing and retrieval tasks. For example, if $k_{\text {in }}$ is defined to be minimum in regions with an homogeneous blue color, the corresponding parameter is the mean value of the blue component within the region. If the segmentation method is applied to a set of RGB images, the parameter values can be stored along with the blue region contours after which the image database can be queried for images containing homogeneous regions with a given blue component value.

D. Examples Of Descriptors ${ }^{18,20}$

1. Constant Intensity

An example of a region descriptor that is region-independent is

$$
k_{\text {in }}(\tau, x)=\phi(\delta-I(x))
$$


where $\phi$ is a smooth, positive, even function increasing on $R^{+}$. This descriptor can be used to segment a region of known intensity $\delta$. If $k_{\text {out }}$ is defined similarly, then $H\left(k_{\text {in }}, k_{\text {out }}\right)$ is equal to zero.

\section{Mean Intensity}

The mean intensity within $\Omega_{\text {in }}(\tau)$ is

$$
\mu(\tau)=\frac{\int_{\Omega_{\text {in }}(\tau)} I(x) \mathrm{d} x}{\int_{\Omega_{\mathrm{in}}(\tau)} \mathrm{d} x} .
$$

If descriptor $k_{\text {in }}$ is $\phi(\mu(\tau)-I(x))$ and $k_{\text {out }}$ is equal to zero, then

$$
H\left(k_{\text {in }}, k_{\text {out }}\right)(\tau, x)=-\frac{\mu(\tau)-I(x)}{\int_{\Omega_{\text {in }}(\tau)} \mathrm{d} y} \int_{\Omega_{\text {in }}(\tau)} \phi^{\prime}(\mu(\tau)-I(y)) \mathrm{d} y
$$

If $\phi$ is the square function, then $H\left(k_{\text {in }}, k_{\text {out }}\right)$ is equal to zero. ${ }^{13,14}$ This descriptor can be used to segment an homogeneous region of unknown intensity.

\section{Variance}

The variance within $\Omega_{\text {in }}(\tau)$ is

$$
\sigma^{2}(\tau)=\frac{\int_{\Omega_{\mathrm{in}}(\tau)}(\mu(\tau)-I(x))^{2} \mathrm{~d} x}{\int_{\Omega_{\mathrm{in}}(\tau)} \mathrm{d} x}
$$

where $\mu(\tau)$ is defined as in (25). If descriptor $k_{\text {in }}$ is $\phi\left(\sigma^{2}(\tau)\right)$ and $k_{\text {out }}$ is equal to zero, then

$$
H\left(k_{\text {in }}, k_{\text {out }}\right)(\tau, x)=\phi^{\prime}\left(\sigma^{2}(\tau)\right)\left[\sigma^{2}(\tau)-(\mu(\tau)-I(x))^{2}\right]
$$

Note that $H\left(k_{\text {in }}, k_{\text {out }}\right)$ depends on $x$ whereas the descriptor (hence, the criterion) does not. This descriptor can be used to segment a region with low noise or low intensity variation.

\section{Shape Of Reference}

When segmenting a region using appropriate descriptors, it can be useful to constrain the active contour to stay close to a shape of reference. This can be done using the following criterion ${ }^{20}$

$$
J_{2}(\tau)=\int_{\Gamma(\tau)} k_{b}(\tau, x) \mathrm{d} x=\int_{\Gamma(\tau)} \phi\left(d\left(x, \Gamma_{\text {ref }}\right)\right) \mathrm{d} x
$$

where $d\left(x, \Gamma_{\text {ref }}\right)$ is the signed distance to $\Gamma_{\text {ref }}$ at $x$, i.e.,

$$
d\left(x, \Gamma_{\text {ref }}\right)=\left\{\begin{array}{l}
+|x-y(x)| \text { if } x \text { is outside } \Gamma_{\text {ref }} \\
-|x-y(x)| \text { otherwise }
\end{array}\right.
$$

where $y(x)$ is the closest point to $x$ belonging to $\Gamma_{\text {ref }}$, and $\phi$ is a smooth, positive, even function increasing on $R^{+}$. In criterion (13), boundary descriptor $k_{b}$ does not depend 
on $\tau$, i.e., on the boundary. Computation of the derivative of boundary-dependent descriptor (29) is different from the developments mentioned in Subsection 2.B. It can be shown ${ }^{20}$ that the derivative is

$$
J_{2}^{\prime}(\tau)=-\int_{\Gamma(\tau)}\left(\phi^{\prime}(d) \boldsymbol{N}_{\mathrm{ref}} \cdot \boldsymbol{N}+\phi(d) \kappa\right) \boldsymbol{v} \cdot \boldsymbol{N} \mathrm{d} x
$$

where $d$ is a short notation for $d\left(x, \Gamma_{\text {ref }}\right)$ and $\boldsymbol{N}_{\text {ref }}$ is the inward unit normal to $\Gamma_{\text {ref }}$ at $y(x)$. The deformation between the shape of reference and the segmentation contour is a free-form deformation (i.e., deformation with unlimited degrees of freedom) as opposed to a parametric transform ${ }^{21,22}$ (i.e., global deformation with few degrees of freedom, e.g., a combination of a translation, a rotation, and a scaling). Although the derivatives of the criteria may be similar in both cases, the parametric transform approach tends to turn the segmentation problem into an iterative best fit search among the set of contours obtained by parametric transform.

\section{Active Contour Implementation}

\section{A. From Parametric To Implicit}

The first implementations of the active contour technique were based on a parametric, or explicit, description of the contour. ${ }^{1}$ It corresponds to a Lagrangian approach. However, management of the evolution, particularly topology changes and sampling density along the contour, is not simple. ${ }^{23}$ Instead, an Eulerian approach known as the level set technique ${ }^{24,25}$ can be used. In two dimensions, the contour is implicitly represented as the intersection of a surface $u=f(x)$, where $x \in D$ and elevation $f$ is a continuous function with positive and negative values, with the plane of elevation zero. The contour can also be seen as the isocontour of level zero on the surface. In three dimensions, the contour is the isosurface of level zero in a volume. In $n$ dimensions, the contour is the hyperplane of level zero with the space filled in with the values of a real, continuous function. Note that the contour can actually be composed of several closed contours without intersections with each other. By a continuous change of function $f$, a contour can appear or disappear without explicit handling. Unfortunately, even with the narrow band implementation, ${ }^{26,27}$ the level set technique has a rather high computational cost and extension of the velocity to levels other than the zero level is not straightforward ${ }^{28,29}$ while it is theoretically necessary. Moreover, a curvature term (minimum length penalty) is usually added to the velocity expression in order to decrease the influence of image noise on the evolution. However, the curvature being a second derivative term, its numerical approximation is usually not accurate. Similarly, the level set gradient (equivalent to the isocontour normal) is numerically approximated. The fast marching technique ${ }^{30}$ is another Eulerian approach to the active contour representation. It could be seen as the limit case where the narrow band is one grid element thick. This technique is much faster than the level set technique. However it can be applied only if one can guarantee that the velocity expression keeps the same sign during evolution. 


\section{B. Splines: Back To The Parametric Approach}

A cubic B-spline has several interesting properties: It is a $C^{2}$ curve, ${ }^{31}$ it is an interpolation curve that minimizes a term close to the square curvature, ${ }^{32}$ and it has an analytical equation (depending on control points) between each pair of consecutive sampling points $\left(p_{i}, p_{i+1}\right)$. It minimizes the following criterion with the constraint that it passes through the sampling points

$$
J_{\text {CubicS }}(\Gamma)=\int_{\Gamma} \Gamma^{\prime \prime}(x)^{2} \mathrm{~d} x
$$

where $\Gamma$ " is the second derivative of $\Gamma$ with respect to the arc length parameter. Note that the sampling points correspond to the ends, or knots, of the spline segments. The velocity has to be computed at the sampling points only. If the sampling is regular, the normal and the curvature can be computed using an exact, fast, recursive filtering algorithm applied to the control points. Therefore, the spline implementation is much less time consuming than the level set technique. ${ }^{33,34}$ Moreover, the minimum curvature-type term property helps in decreasing the influence of noise without the need to add a curvature term to the velocity. Nevertheless, noise in the image still implies noise in the velocity which, if sampling is fine, usually leads to an irregular contour because, despite the smooth curvature property, a cubic B-spline is an interpolation curve. Finally, let us recall that, unlike with level sets, management of topology changes has to be performed explicitly and is not an easy task. Also note that the number of sampling points is a parameter. Less sampling points implies a curve more rigid, or smoother, preventing from segmenting regions with a high curvature.

\section{Smoothing Splines}

A smoothing spline is an approximation curve controlled by a parameter balancing the trade-off between interpolation error and smoothness. ${ }^{35,36}$ It minimizes the following criterion

$$
J_{\text {SmoothS }}(\Gamma)=\lambda \int_{\Gamma} \Gamma^{\prime \prime}(x)^{2} \mathrm{~d} x+\sum_{i}\left(p_{i}-\Gamma_{i}\right)^{2}
$$

where $\lambda$ is a parameter, $p_{i}$ is a sampling point, and $\Gamma_{i}$ is a knot. Note that knots do not correspond to sampling points anymore. The smoothing spline is only constrained to be confined to a "band" surrounding the sampling points. The smaller parameter $\lambda$, the narrower the band. When $\lambda$ is equal to zero, the smoothing spline is a classical interpolation spline. Thus, if sampling is fine and noise is high, the smoothing spline can still be smooth. As with cubic B-splines, normal and curvature can be computed exactly and efficiently.

\section{Examples Of Applications}

The following results were obtained using the level set technique except otherwise noted. 


\section{A. Image Segmentation}

\section{Region Competition}

Image "X-ray" is a $512 \times 512$, greyscale image. Descriptor $k_{\text {in }}$ and $k_{\text {out }}$ were both variance descriptors (see Subsection 2.D.3). This type of segmentation is called a region competition. To give a brief comparison in terms of computation time between the level set technique and the smoothing spline implementation, the image was segmented using both algorithms. See Fig. 1. The segmentation using level sets was
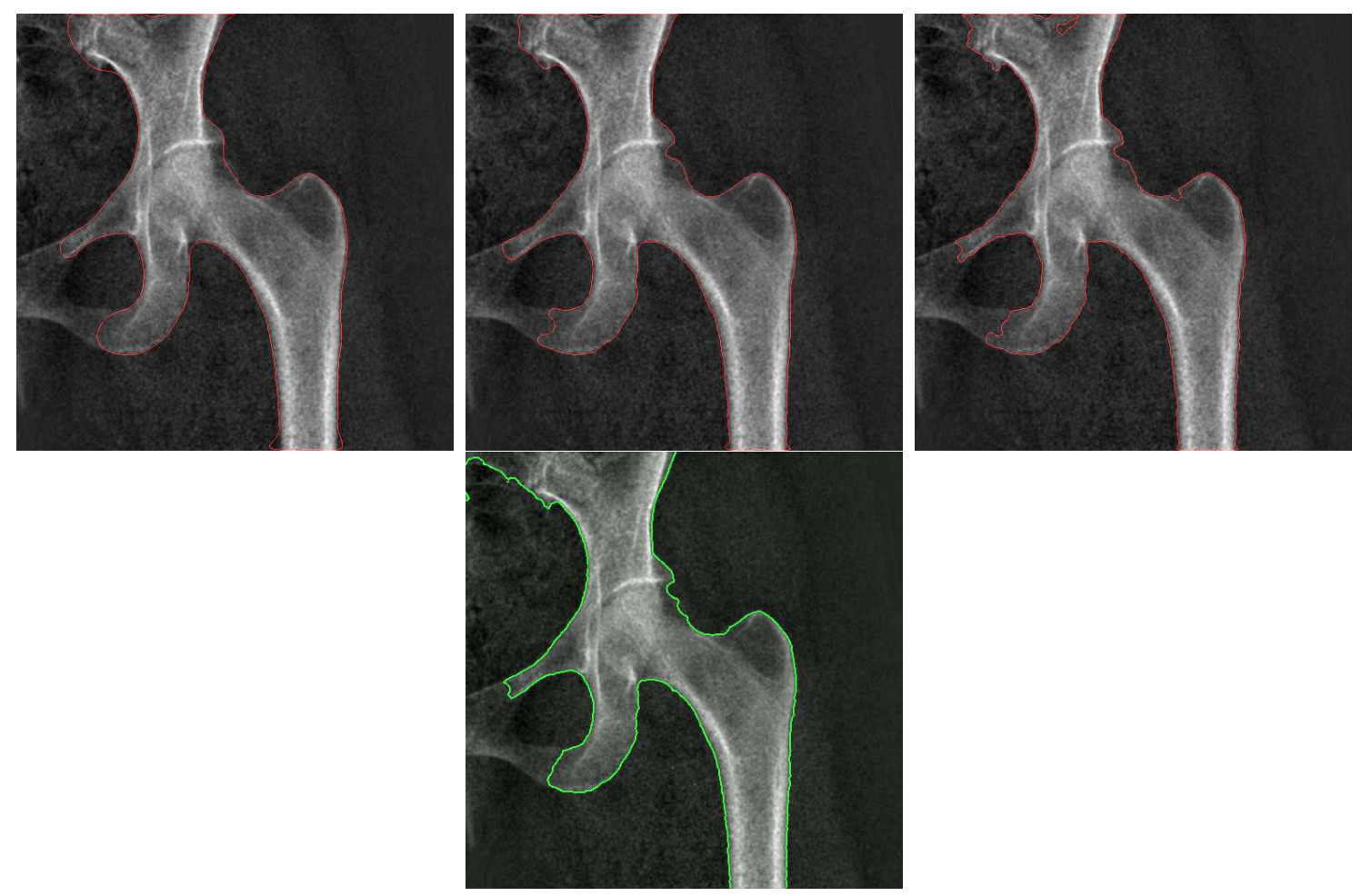

Fig. 1. Segmentation of "X-ray": Segmentation using a smoothing spline with 128 sampling points (top left), with 256 sampling points (top middle), and with 512 sampling points (top right) and $\lambda$ equal to 0.005; Segmentation using level sets (bottom).

performed roughly 15 times slower than the segmentation using a smoothing spline with 256 sampling points. With 128 sampling points the ratio increases to 22 times. However the segmentation contour is less accurate because it is too smooth compared to the shape of the object of interest. With 512 sampling points the ratio decreases to 6.5 times and the segmentation contour is not smooth enough. The influence of parameter $\lambda$ can be seen on Fig. 2. The segmentation contour is smoother with a higher $\lambda$. 

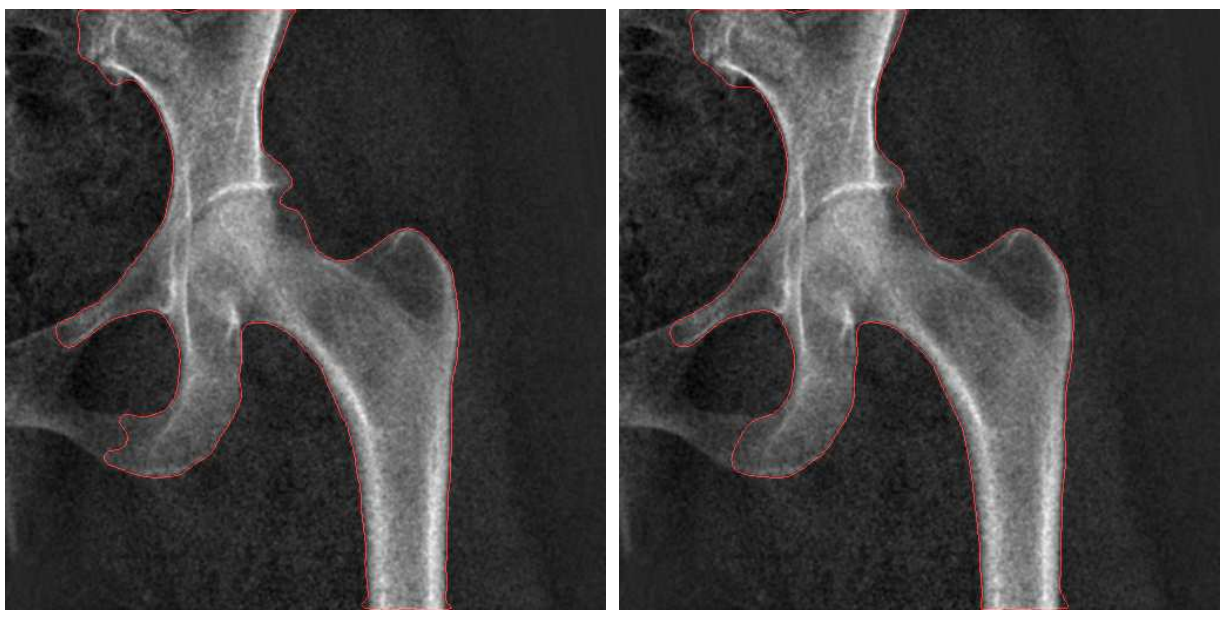

Fig. 2. Segmentation of "X-ray": Segmentation using a smoothing spline with 256 sampling points and $\lambda$ equal to: 0.005 (left) and 0.025 (right).

\section{Shape Of Reference}

Image "Phone" is a $352 \times 288$, color image. Two descriptors were used for the segmentation: A variance descriptor (see Subsection 2.D.3) for the face $\left(k_{\text {in }} /\right.$ statistical descriptor) and a shape of reference descriptor (see Subsection 2.D.4) as a constraint ( $k_{b} /$ geometrical descriptor). Descriptor $k_{\text {out }}$ was taken equal to zero. Combination of these descriptors implies a competition between the shape prior and the statistical information of the object to be segmented. If the shape of reference constraint is omitted, the face segmentation includes part of the hand of the character and does not include the lips. A shape of reference was heuristically defined allowing to segment accurately the face. See Fig. 3.
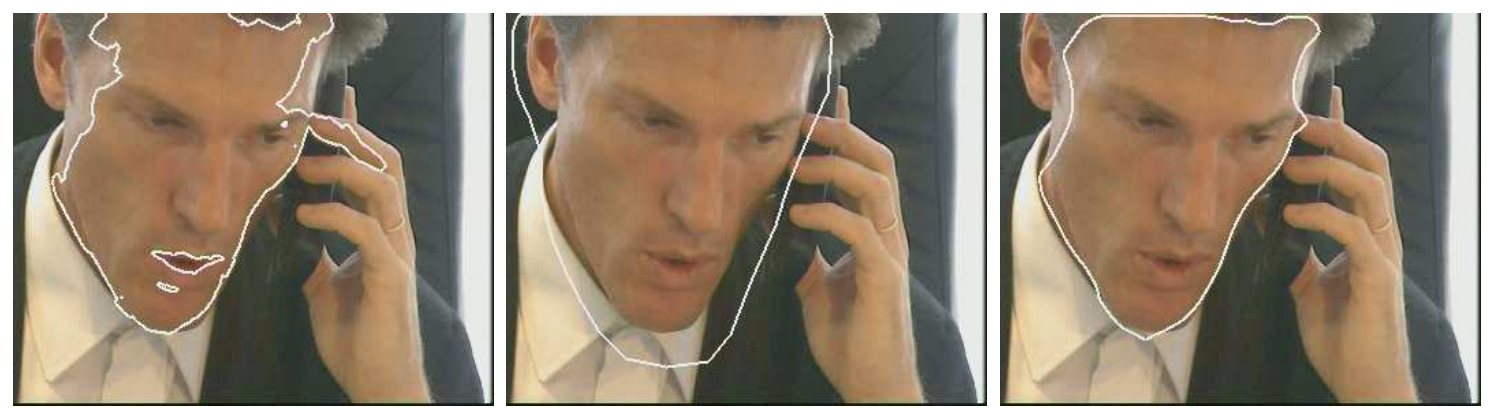

Fig. 3. Segmentation of "Phone": Segmentation without shape of reference (left); Shape of reference (middle); Segmentation with the shape of reference constraint (right). 


\section{B. Sequence Segmentation}

Sequence "Akiyo" is composed of three hundred $352 \times 288$, color images. Descriptor $k_{\text {in }}$ was taken equal to a constant penalty. Descriptor $k_{\text {out }}$ was defined as the difference between the current image and a robust estimate of the background image computed using the $n_{b}$ previous images ${ }^{37}\left(n_{b}=20\right)$, or less for the first $n_{b}$ images. This descriptor takes advantage of the temporal information of the sequence: It is a motion detector. In case of a moving background due to camera motion, a mosaicking technique can be used to estimate the background image. ${ }^{38}$ Descriptor $k_{\text {out }}$ was taken equal to zero. The first image of the sequence was not segmented since no previous images were available to estimate the background. See Fig. 4 and 5.
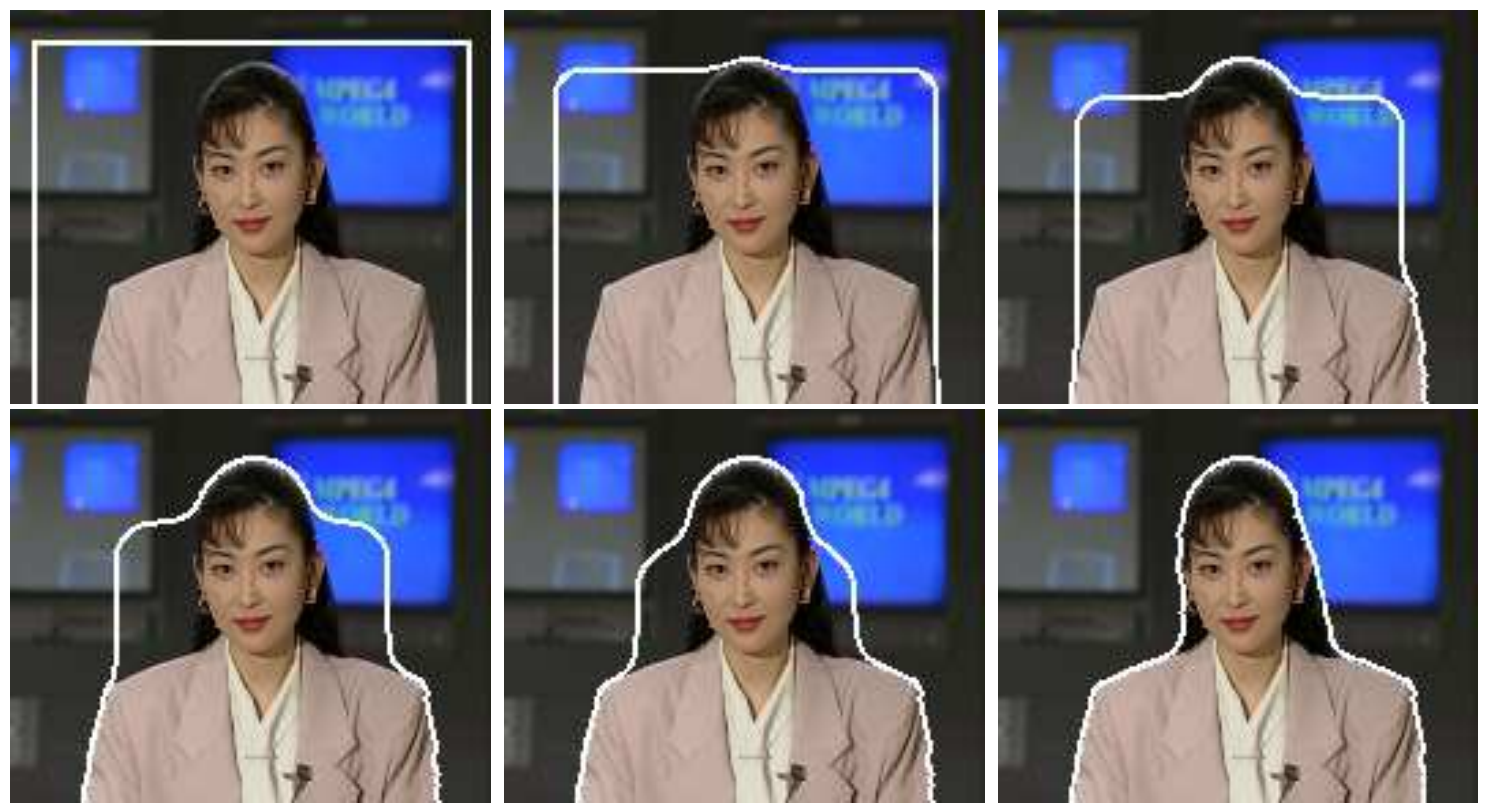

Fig. 4. Segmentation of Akiyo: Image 2, evolution from the initial contour until convergence.

\section{Tracking}

Sequence "Erik" is composed of fifty $256 \times 256$, color images. Given a segmentation of Erik's face in the first image, the purpose was to track the face throughout the sequence using the segmentation of the previous image to constrain the segmentation of the current frame. ${ }^{20}$ See Fig. 6. Two descriptors were used: A variance descriptor (see Subsection 2.D.3) for the face ( $k_{\text {in }} /$ statistical descriptor) and a shape of reference descriptor (see Subsection 2.D.4) as a constraint ( $k_{b} /$ geometrical descriptor). Descriptor $k_{\text {out }}$ was taken equal to zero. The shape of reference in the current image was defined as an affine transform (i.e., a combination of a translation, a rotation, and a scaling) of the segmentation contour in the previous image. This transform can be interpreted as the global motion of the object of interest between the previous and 

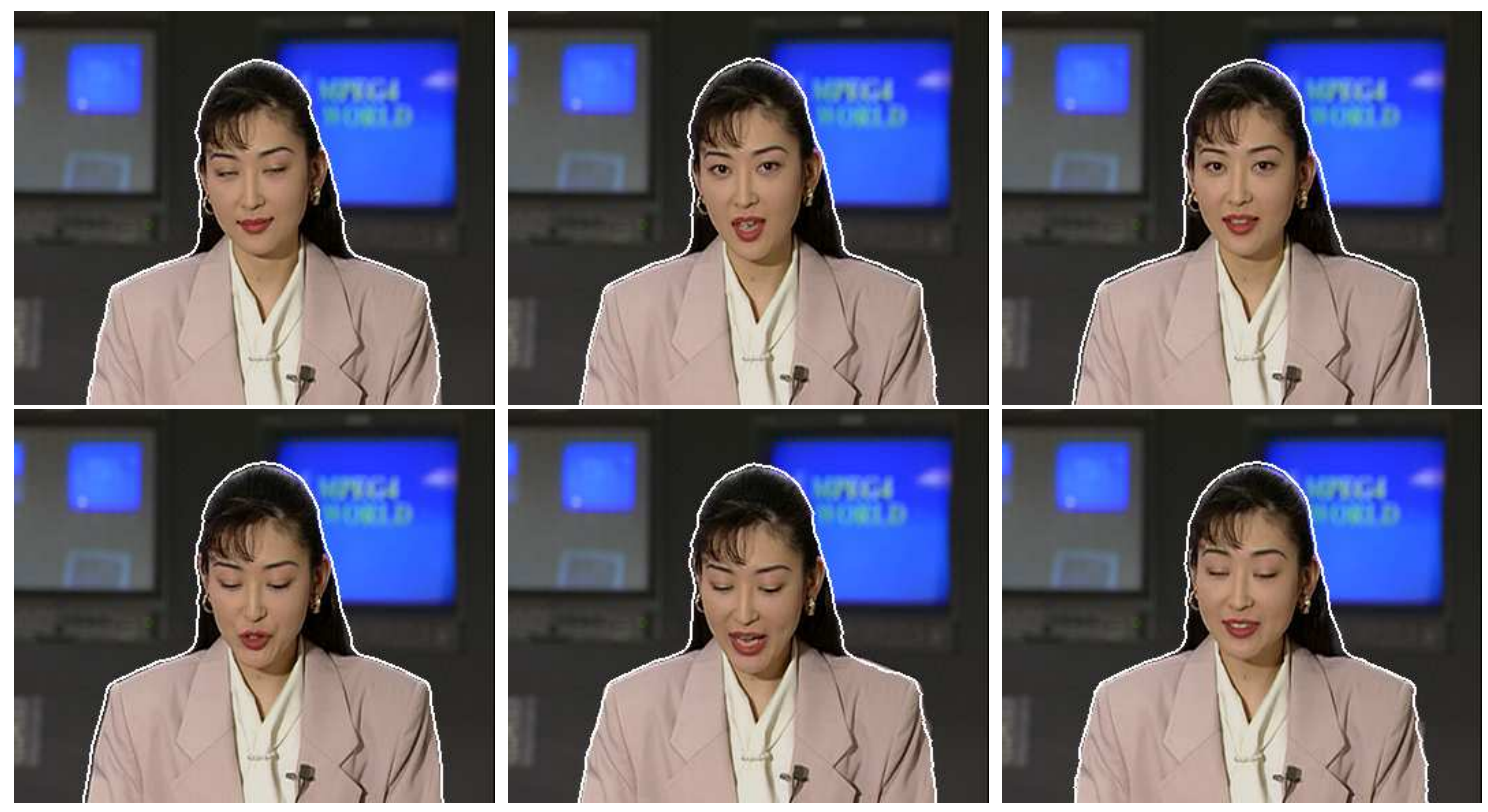

Fig. 5. Segmentation of Akiyo: Images 5, 15, 25, 40, 55, and 70 out of 300.

the current image. It was estimated by a block matching method with the Zero-mean Normalized Sum of Squared Differences (ZNSSD) criterion applied to the points of the segmentation contour in the previous image in order to find their corresponding points in the current image. The resulting contour was used both as the shape of reference and the initial contour of the active contour process for segmentation of the current image. As a consequence, the segmentation contour is a free-form deformation of the contour resulting from the global motion estimation. Other choices can be made for separation of the overall motion from the deformation. ${ }^{39}$

\section{Acknowledgments}

Image "Phone" was provided by france telecom, France. The X-ray image was provided by Diagnostic Medical Systems (DMS), Montpellier, France.

\section{Appendix}

Applying theorem 1 with $\Omega_{\text {in }}(\tau), k_{\text {in }}$ and $D=\Omega_{\text {in }}(\tau)$ for the first integral of $(15)$ and with $\Omega_{\text {out }}(\tau), k_{\text {out }}$ and $D=\Omega_{\text {out }}(\tau)$ for the second integral, we have

$$
\begin{aligned}
\tilde{J}_{1}^{\prime}(\tau)= & \int_{\Omega_{\text {in }}(\tau)} \frac{\partial k_{\text {in }}}{\partial \tau} \mathrm{d} x-\int_{\Gamma(\tau)} \boldsymbol{v} \cdot \boldsymbol{N} k_{\text {in }} \mathrm{d} x+\int_{\Omega_{\text {out }}(\tau)} \frac{\partial k_{\text {out }}}{\partial \tau} \mathrm{d} x- \\
& \int_{\partial \Omega_{\text {out }}(\tau)} \boldsymbol{w} \cdot \boldsymbol{N}_{\Omega_{\text {out }}} k_{\text {out }} \mathrm{d} x .
\end{aligned}
$$

Boundary $\partial \Omega_{\text {out }}(\tau)$ is the union of $\partial D$ and $\Gamma(\tau)$. Note that the velocity of $\Gamma(\tau)$ is identical whether it is seen from $\Omega_{\text {in }}(\tau)$ or $\Omega_{\text {out }}(\tau)$. On the other hand, the normal is 


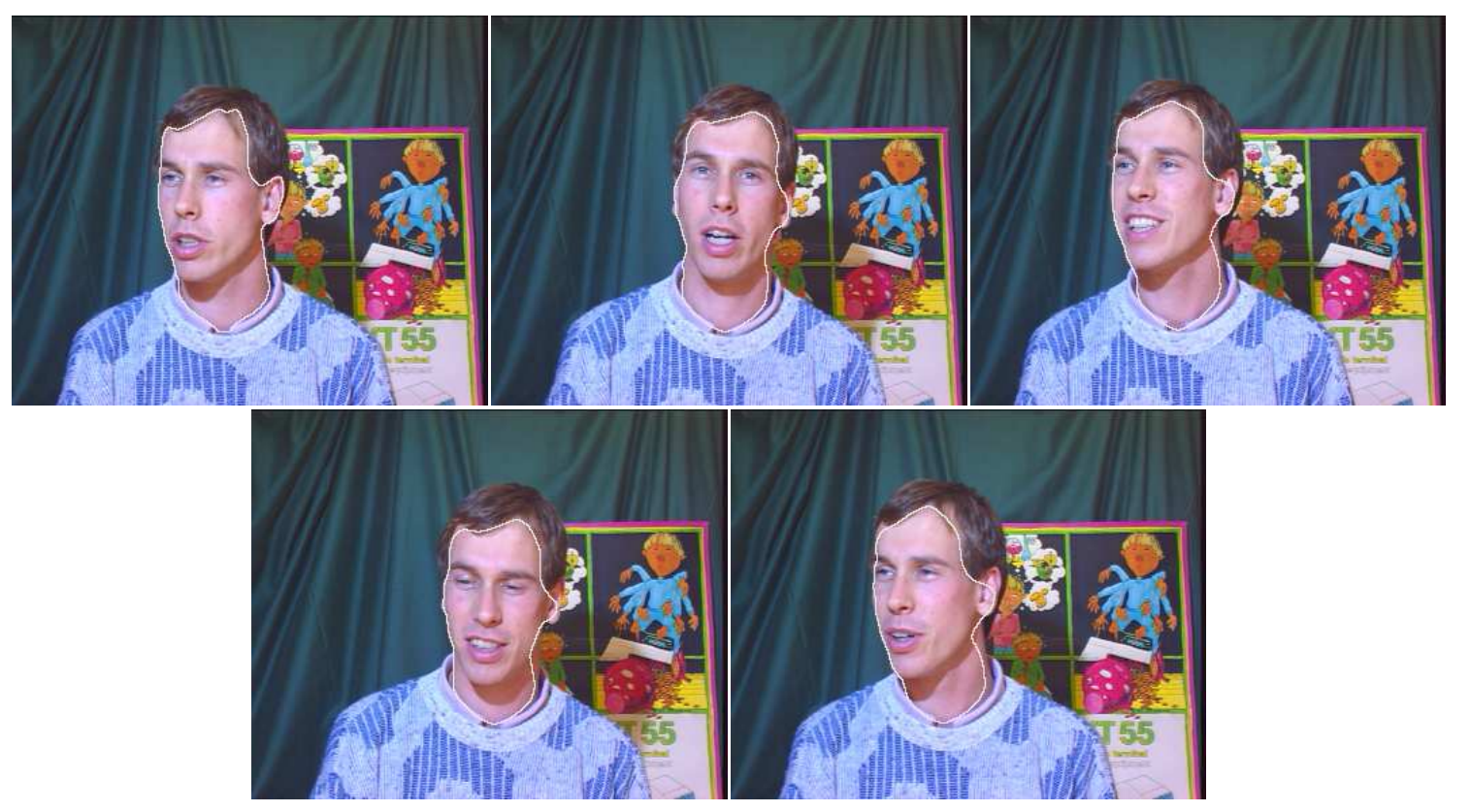

Fig. 6. Tracking of Erik's face: Images 1, 10, 27, 40, and 50 out of 50.

opposite. Therefore

$$
\begin{aligned}
\tilde{J}_{1}^{\prime}(\tau)= & \int_{\Omega_{\text {in }}(\tau)} \frac{\partial k_{\text {in }}}{\partial \tau} \mathrm{d} x-\int_{\Gamma(\tau)} \boldsymbol{v} \cdot \boldsymbol{N} k_{\text {in }} \mathrm{d} x+ \\
& \int_{\Omega_{\text {out }}(\tau)} \frac{\partial k_{\text {out }}}{\partial \tau} \mathrm{d} x-\int_{\Gamma(\tau)} \boldsymbol{v} \cdot(-\boldsymbol{N}) k_{\text {out }} \mathrm{d} x- \\
& \int_{\partial \Omega_{\text {out }}(\tau) \backslash \Gamma(\tau)} \boldsymbol{w} \cdot \boldsymbol{N}_{\Omega_{\text {out }}} k_{\text {out }} \mathrm{d} x \\
= & \int_{\Omega_{\text {in }}(\tau)} \frac{\partial k_{\text {in }}}{\partial \tau} \mathrm{d} x-\int_{\Gamma(\tau)} \boldsymbol{v} \cdot \boldsymbol{N}\left(k_{\text {in }}-k_{\text {out }}\right) \mathrm{d} x+ \\
& \int_{\Omega_{\text {out }}(\tau)} \frac{\partial k_{\text {out }}}{\partial \tau} \mathrm{d} x-\int_{\partial \Omega_{\text {out }}(\tau) \backslash \Gamma(\tau)} \boldsymbol{w} \cdot \boldsymbol{N}_{\Omega_{\text {out }}} k_{\text {out }} \mathrm{d} x
\end{aligned}
$$


Let $K$ be the function equal to $k_{\text {in }}$ in $\Omega_{\text {in }}(\tau)$ and $k_{\text {out }}$ in $\Omega_{\text {out }}(\tau)$ and let $[[K]]$ be the jump of $K$ across $\Gamma(\tau)$ : [[K]] $(x)=k_{\text {in }}(x)-k_{\text {out }}(x)$ for $x \in \Gamma(\tau)$. Finally

$$
\begin{aligned}
\tilde{J}_{1}^{\prime}(\tau)= & \int_{\Omega_{\text {in }}(\tau)} \frac{\partial k_{\text {in }}}{\partial \tau} \mathrm{d} x+\int_{\Omega_{\text {out }}(\tau)} \frac{\partial k_{\text {out }}}{\partial \tau} \mathrm{d} x- \\
& \int_{\Gamma(\tau)} \boldsymbol{v} \cdot \boldsymbol{N}\left(k_{\text {in }}-k_{\text {out }}\right) \mathrm{d} x-\int_{\partial \Omega_{\text {out }}(\tau) \backslash \Gamma(\tau)} \boldsymbol{w} \cdot \boldsymbol{N}_{\Omega_{\text {out }}} k_{\text {out }} \mathrm{d} x \\
= & \int_{\Omega_{\text {in }}(\tau)} \frac{\partial K}{\partial \tau} \mathrm{d} x+\int_{\Omega_{\text {out }}(\tau)} \frac{\partial K}{\partial \tau} \mathrm{d} x- \\
& \int_{\Gamma(\tau)} \boldsymbol{v} \cdot \boldsymbol{N}[[K]] \mathrm{d} x-\int_{\partial \Omega_{\text {out }}(\tau) \backslash \Gamma(\tau)} \boldsymbol{w} \cdot \boldsymbol{N}_{\Omega_{\text {out }}} K \mathrm{~d} x \\
= & \int_{D} \frac{\partial K}{\partial \tau} \mathrm{d} x-\int_{\Gamma(\tau)} \boldsymbol{v} \cdot \boldsymbol{N}[[K]] \mathrm{d} x-\int_{\partial \Omega_{\text {out }}(\tau) \backslash \Gamma(\tau)} \boldsymbol{w} \cdot \boldsymbol{N}_{\Omega_{\text {out }}} K \mathrm{~d} x
\end{aligned}
$$

\section{References}

1. M. Kass, A. Witkin, and D. Terzopoulos, "Snakes: Active contour models," International Journal of Computer Vision, vol. 1, 321-332 (1988).

2. L. Cohen, "On active contour models and balloons," Computer Vision, Graphics and Image Processing: Image Understanding, vol. 53, 211-218 (1991).

3. V. Caselles, R. Kimmel, and G. Sapiro, "Geodesic active contours," International Journal of Computer Vision, vol. 22, 61-79 (1997).

4. L. Cohen, E. Bardinet, and N. Ayache, "Reconstruction of digital terrain model with a lake," in Conference on Geometric Methods in Computer Vision II, Proc. SPIE 2031, 38-50 (1993).

5. R. Ronfard, "Region-based strategies for active contour models," International Journal of Computer Vision, vol. 13, 229-251 (1994).

6. A. Chakraborty, L. Staib, and J. Duncan, "Deformable boundary finding in medical images by integrating gradient and region information," IEEE Transactions on Medical Imaging, vol. 15, 859-870 (1996).

7. S. Zhu and A. Yuille, "Region competition: unifying snakes, region growing, and bayes/MDL for multiband image segmentation," IEEE Transactions on Pattern Analysis and Machine Intelligence, vol. 18, 884-900 (1996).

8. N. Paragios and R. Deriche, "Geodesic active regions for motion estimation and tracking," in Proceedings of International Conference on Computer Vision, (1999), pp. 688-694.

9. N. Paragios and R. Deriche, "Geodesic active regions and level set methods for supervised texture segmentation," International Journal of Computer Vision, vol. 46, 223-247 (2002).

10. A. J. Yezzi, A. Tsai, and A. Willsky, "A statistical approach to snakes for bimodal and trimodal imagery," in Proceedings of International Conference on Computer Vision, (1999), pp. 898-903.

11. C. Chesnaud, P. Réfrégier, and V. Boulet, "Statistical region snake-based segmentation adapted to different physical noise models," IEEE Transactions on 
Pattern Analysis and Machine Intelligence, vol. 21, 1145-1156 (1999).

12. C. Samson, L. Blanc-Féraud, G. Aubert, and J. Zerubia, "A level set model for image classification," International Journal of Computer Vision, vol. 40, 187-197 (2000).

13. T. Chan and L. Vese, "Active contours without edges," IEEE Transactions on Image Processing, vol. 10, 266-277 (2001).

14. E. Debreuve, M. Barlaud, G. Aubert, and J. Darcourt, "Space time segmentation using level set active contours applied to myocardial gated SPECT," IEEE Transactions on Medical Imaging, vol. 20, 643-659 (2001).

15. O. Amadieu, E. Debreuve, M. Barlaud, and G. Aubert, "Inward and outward curve evolution using level set method," in Proceedings of International Conference on Image Processing, (1999), pp. 188-192.

16. J. Sokolowski and J.-P. Zolésio, Introduction to shape optimization: Shape sensitivity analysis (Springer-Verlag, Berlin, 1992).

17. M. C. Delfour and J.-P. Zolésio, Shapes and geometries: Analysis, Differential Calculus and Optimization (Society for Industrial and Applied Mathematics, Philadelphia, 2001).

18. S. Jehan-Besson, M. Barlaud, and G. Aubert, "DREAM ${ }^{2} \mathrm{~S}$ : Deformable regions driven by an eulerian accurate minimization method for image and video segmentation," International Journal of Computer Vision, vol. 53, 45-70 (2003).

19. G. Aubert, M. Barlaud, O. Faugeras, and S. Jehan-Besson, "Image segmentation using active contours: Calculus of variations or shape gradients?," SIAM Journal of Applied Mathematics, vol. 63, 2128-2154 (2003).

20. M. Gastaud, M. Barlaud, and G. Aubert, "Tracking video objects using active contours," in Proceedings of Workshop on Motion and Video Computing, (2002), pp. 90-95.

21. Y. Chen, H. D. Tagare, S. Thiruvenkadam, F. Huang, D. Wilson, K. S. Gopinath, R. W. Briggs, and E. A. Geiser, "Using prior shapes in geometric active contours in a variational framework," International Journal of Computer Vision, vol. 50, 315-328 (2002).

22. D. Cremers, F. Tischhäuser, J. Weickert, and C. Schnörr, "Diffusion snakes: Introducing statistical shape knowledge into the mumford-shah functional," International Journal of Computer Vision, vol. 50, 295-313 (2002).

23. P. Charbonnier and O. Cuisenaire, "Une étude des contours actifs : modèles classique, géométrique et géodésique," Technical Report 163, Laboratoire de télécommunications et télédétection, Université catholique de Louvain, 1996.

24. S. Osher and J. A. Sethian, "Fronts propagating with curvature-dependent speed: Algorithms based on hamilton-jacobi formulations," Journal of Computational Physics, vol. 79, 12-49 (1988).

25. G. Barles, "Remarks on a flame propagation model," Technical Report 464, Projet Sinus, Institut National de Recherche en Informatique et en Automatique (INRIA) de Sophia Antipolis, Sophia Antipolis, France, 1985.

26. D. L. Chopp, "Computing minimal surfaces via level set curvature flow," Journal of Computational Physics, vol. 106, 77-91 (1993). 
27. D. Adalsteinsson and J. A. Sethian, "A fast level set method for propagating interfaces," Journal of Computational Physics, vol. 118, 269-277 (1995).

28. D. Adalsteinsson and J. A. Sethian, "The fast construction of extension velocities in level set methods," Journal of Computational Physics, vol. 148, 2-22 (1999).

29. J. Gomes and O. D. Faugeras, "Reconciling distance functions and level sets," Journal of Visual Communication and Image Representation, vol. 11, 209-223 (2000).

30. J. A. Sethian, "A fast marching level set method for monotonically advancing fronts," in Proceedings of the National Academy of Sciences, (1996), vol. 93, pp. $1591-1595$.

31. P. Thevenaz, T. Blu, and M. Unser, "Interpolation revisited [medical images application]," IEEE Transactions on Medical Imaging, vol. 19, 739-758 (2000).

32. M. Jacob, T. Blu, and M. Unser, "A unifying approach and interface for splinebased snakes," in International Symposium on Medical Imaging: Image Processing, Proc. SPIE 4322, 340-347 (2001).

33. F. Precioso and M. Barlaud, "B-spline active contours with handling of topological changes for fast video segmentation," EURASIP Journal on Applied Signal Processing, Special issue on Image Analysis for Multimedia Interactive Services Part II, vol. 2002, 555-560 (2002).

34. F. Precioso and M. Barlaud, "Regular spatial B-spline active contours for fast video segmentation," in Proceedings of International Conference on Image Processing, (2002), pp. 761-764.

35. M. Unser, A. Aldroubi, and M. Eden, "B-spline signal processing: Part I-theory," IEEE Transactions on Signal Processing, vol. 41, 821-833 (1993).

36. F. Precioso, M. Barlaud, T. Blu, and M. Unser, "Smoothing B-spline active contour for fast and robust image and video segmentation," in Proceedings of International Conference on Image Processing, (2003), pp. I-137-I-140.

37. S. Jehan-Besson, M. Barlaud, and G. Aubert, "Video object segmentation using eulerian region-based active contours," in Proceedings of International Conference on Computer Vision, (2001), pp. 353-361.

38. M. Gastaud and M. Barlaud, "Video segmentation using region based active contours on a group of pictures," in Proceedings of International Conference on Image Processing, (2002), vol. II, pp. 81-84.

39. S. Soatto and A. J. Yezzi, "Deformotion: Deforming motion, shape average and the joint registration and segmentation of images," in Proceedings of European Conference on Computer Vision, (2002), pp. 32-47. 